\title{
Transport networks
}

\author{
Dr. César Ducruet \\ Centre National de la Recherche Scientifique (CNRS) \\ cdu@parisgeo.cnrs.fr
}

Pre-final version of the entry published in Richardson D. et al. (Eds.) (2017) The International Encyclopedia of Geography, Wiley-Blackwell \& Association of American Geographers, https://doi.org/10.1002/9781118786352.wbieg0024

\begin{abstract}
A transport network is basically defined as a set of connected nodes and links allowing the circulation of goods and/or individuals. Traditionally studied for itself and in rather abstract ways through graph-theoretical and economic lenses, the transport network has increasingly been defined as one component only, albeit crucial, of wider logistical, territorial, and societal realities.
\end{abstract}

Transport networks may be defined as sets of connected nodes and links allowing for the circulation of individuals and/or commodities. Traditionally, transport networks corresponded to physical infrastructure such as roads, railways, canals and rivers, streets and subway lines, while the concept also includes airline and maritime networks in which nodes are (sea)ports and airports, as well as telecommunications networks transmitting information and knowledge flows, such as telephone lines, the Internet, and parent technologies (e.g. telegraph, mobile phones). In fact, the definition of transport networks can be extended to any support of circulation, as natural scientists analyze, for instance, termite mounds and fungal networks just like geographers and engineers study road networks. Tero et al. (2010) have particularly demonstrated that biological networks such as those built by slime molds are "with comparable efficiency, fault tolerance, and cost to those of real-world infrastructure networks - in this case, the Tokyo rail system". While the nature of nodes may vary from one network to the other, those are often crossings, stations, terminals, cities or countries etc., while links can be of totally different nature, from physically grounded tracks to more ephemeral flows. In all cases, transport 
networks belong to the category of "spatial networks" that comprises many other networks such as so-called technical networks (e.g. power grids) and any network being physically (and often geographically) grounded into space (see Barthelemy, 2010). Like any other network and according to the language used by graph theory, a branch of mathematics describing the topological properties of networks, a transport network may be planar or non-planar (i.e. without or with crossings between links or "edges"), directed or undirected, weighted or not (i.e. where links carry a certain quantity e.g. of traffic, distance, or time). A multimodal transport network may be understood as a "multigraph" where two given nodes are connected via at least two links of different nature. However, the concept of transport network does not entirely belong to graph theory; it includes other approaches, such as transport history, spatial analysis, economics, or engineering, where nodes, links and flows are considered by means of other methods, such as discourses, policies, actors, governance, planning, and dedicated Geographical Information Systems for Transport (GIS-T).

Traditionally in geography, transport networks have been the focus of scholars willing to apply quantitative methods to geographical research. Transport networks and flows received foremost attention in the 1960s by US geographers. They particularly developed the idea of ideal-typical sequences of transport network development and applied several graph-theoretical methods to road and railway networks (Taaffe and Gauthier, 1973). The most renowned model describes six successive phases inspired from the evolution of transport networks in West Africa (see Rodrigue, 2014 for a useful online illustration). In a first phase (Scattered ports), a set of ports along a coastline is characterized by homogeneity in traffic volumes and connection levels based on local resources. The second phase (Penetration lines and port concentration) witnesses an expansion of the hinterland benefitting to only a minority of such ports through new transport 
lines such as rail or canal. The subsequent phases reinforce a path-dependent process by which initially favored ports continue to grow proportionally to the importance of their inland accessibility as the corridor emerges gradually through economies of scale. Although this model has been updated to take into account overseas linkages among ports and more recent trends of de-concentration, many critiques have been addressed given the linear dimension of the successive phases which ultimately always lead to concentration in the core and decline in the periphery. Similar criticism emerged from (trans)port specialists about early urban models, such as the central place theory, due to its inability incorporating deviations caused by specific transport configurations (sea-land, intermodal), as in the works of British geographer James Bird. In the early days however, limited computational power largely explained the reliance upon small, planar networks easily extracted from city and country maps, while detailed - and often more voluminous - data about transport flows remained hardly accessible.

Two central and recurrent themes until nowadays are the topology of transport networks and the accessibility and centrality of their nodes. The topology may refer to the differences between the actual layout of a given transport network and its simplified representation as a graph, i.e. straight lines (or edges) between nodes (or vertices). The graph itself is often understood as a matrix, sometimes called origin-destination (O-D) matrix, where links exist or not between nodes ordered in lines and columns (Figure 1). Several measures express a variety of topological features based on such a graph or matrix (Kansky, 1963), such as the size of the network (number of nodes and links), its density (number of links per node), completeness (proportion of existing links in the maximum possible number of links), cliquishness (number of cycles, transitivity as measured by the average proportion of connected adjacent neighbors in the maximum possible number of connected neighbors), etc. Using the graph or matrix to calculate 
network indices makes it possible to apply any graph-theoretical measure such as those found in Social Network Analysis (SNA) and complex systems, despite fundamental differences in the nature of the studied networks. Some measures may not be interpreted the same depending on if people, firms, terminals, or neurons are concerned. Nevertheless, transport networks are common objects of an emerging "science of networks" notably since physics and computer science started to analyze such networks with renewed tools and concepts since the late 1990s (Newman, 2010). Other aspects of transport networks have thus been explored, such as their scale-free and/or small-world dimensions, the probability for nodes to connect other nodes of comparable size (assortativity), the average shortest path length, the "rich-club" coefficient, etc. While the degree distribution follows a power-law function in scale-free networks, small-world networks are characterized by the existence of dense communities (or subgroups of nodes). The rich-club dimension refers to networks in which nodes with many links connect primarily with each other. Great care must be inferred, however, in comparing different networks via such methods because network indices are often biased by network size. Such measures better apply to the evolution of a single network over time, or to the comparison between actual and optimal configurations. For instance, a given (planar) transport network, if weighted by cost or other metric, is best understood when confronted to its two possible extreme situations, i.e. when all possible links are built (cf. greedy triangulation) or when only the optimal links are retained (cf. minimum cost spanning tree). Novel evidence could have been proposed based on such methods when comparing cities of the world through the configuration of their streets, in terms of planning models, urban architecture, and overall circulation efficiency (see Barthélemy and Flammini, 2009). 


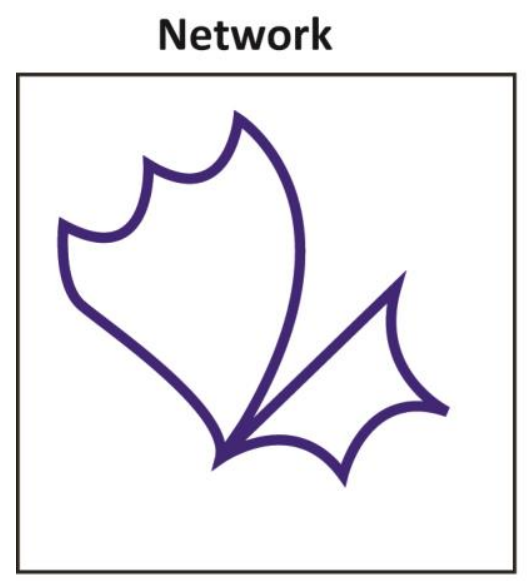

Directed graph

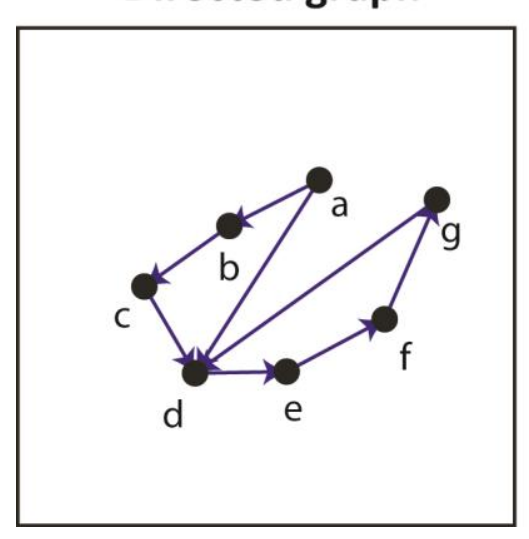

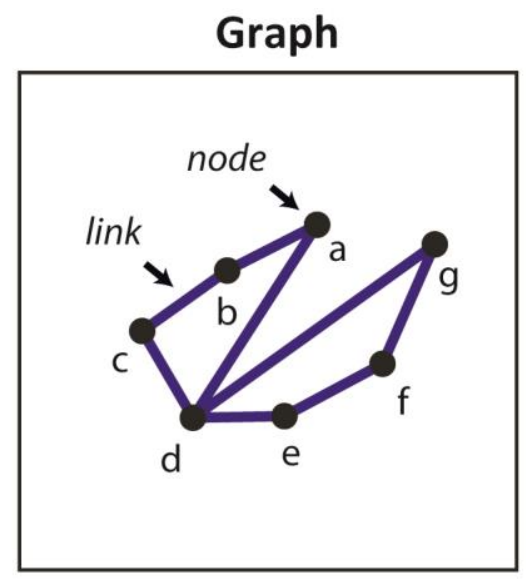

Weighted graph

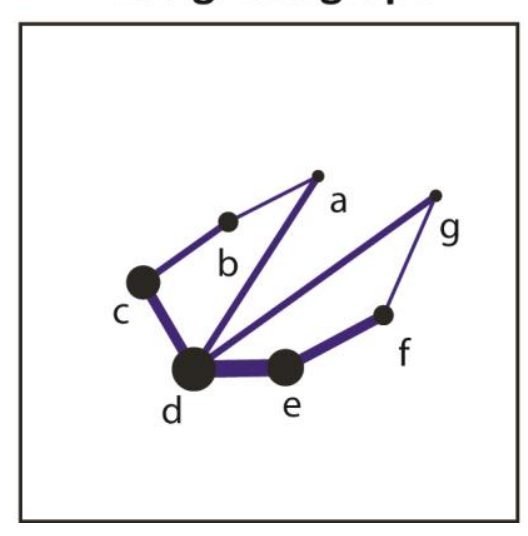

Matrix

\begin{tabular}{|c|c|c|c|c|c|c|c|}
\hline & a & b & c & d & e & f & g \\
\hline a & 0 & 1 & 0 & 1 & 0 & 0 & 0 \\
\hline b & 1 & 0 & 1 & 0 & 0 & 0 & 0 \\
\hline c & 0 & 1 & 0 & 1 & 0 & 0 & 0 \\
\hline d & d & 0 & 1 & 0 & 1 & 0 & 1 \\
\hline e & 0 & 0 & 0 & 1 & 0 & 1 & 0 \\
\hline f & 0 & 0 & 0 & 0 & 1 & 0 & 1 \\
\hline g & 0 & 0 & 0 & 1 & 0 & 1 & 0 \\
\hline
\end{tabular}

\section{Multigraph}

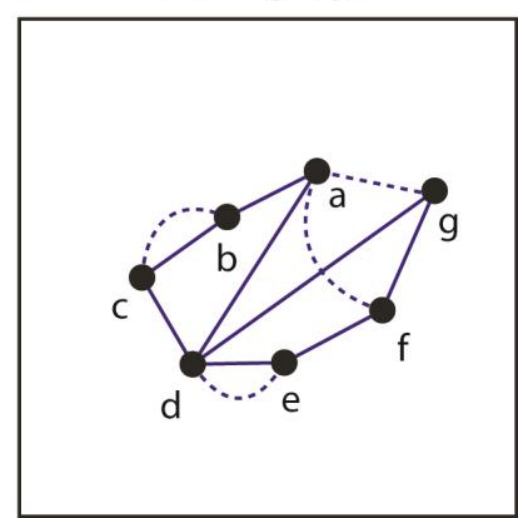

Figure 1: Representations of a transport network

At the node level, measures are similar and may look at the immediate environment of nodes by the number of adjacent neighbors (degree centrality), which correspond to the number of connected airports for instance. In a road or railway network, cities or stations can be compared according to their farness (or eccentricity) to other nodes, their occurrence on shortest paths, etc. In a weighted network, it is also interesting to look at the proportion of traffic depending on the biggest link; in the case of maritime transport, it revealed the extent to which certain ports heavily depended on a close competitor despite their high traffic volume (e.g.

Shenzhen vs. Hong Kong). Are larger nodes more central in the transport network? What are the respective roles of local and global centralities? Certain findings could have pointed at 
interesting functions of some nodes, as in an air transport network, some nodes despite having a few links can still be highly accessible, due to their bridge role between large regions, while larger and more connected nodes often connect across farther topological and kilometric distances. In turn, the existence of such "regions" in a transport network is revealed by means of various techniques of clustering nodes based on certain attributes of connectivity. Are best connected airports or seaports geographically close? How can commuting flows help delineating functional urban areas and/or urban regions? Clustering algorithms applied to transport networks, such as modularity, have, among other results, verified the underlying regional patterns of global airline and maritime flows. Single linkage analysis, for instance, was applied to telecommunication flows as early as in the 1960 s by US geographers in order to delineate socalled "nodal regions" in a given urban system.

Complementary methods of network coupling based on the concept of multigraph have explored how different transport networks share similarities but also vulnerabilities. This is where the network analysis of transport meets more practical problems of disruptions, rerouting and congestion, but also optimization. Where should a new node or link be placed in order to make the transport network(s) more robust to all kinds of failures and targeted attacks? How should two or more transport networks connected so as to limit such shocks? Such questions are particularly relevant in the case of networks being largely dependent upon a few large, critical nodes (e.g. Suez and Panama canals for sea transport). Other aspects to be explored via coupled networks include the study of potential intermodal shifts, the co-evolution of transport networks, the multimodal accessibility of cities, among others. Geographers have been particularly keen in analyzing transport network dynamics under various contexts, such as the impact of deregulation on airline networks, of railway development on urban accessibility, etc. Notably, Bogart (2009) 
is a good example of a study combining a dynamical and a multimodal approach when looking at the evolution of canals, roads, and ports during the English Industrial Revolution. This work notably demonstrated the positive effect of road development on canal development, but a less significant role of ports probably due to their earlier existence. It also suggested that investing in a canal would be less profitable when nearby road improvements were initiated due to higher uncertainties about potential profits from canal tolls.

Overall, graph-theoretical approaches to transport networks attracted a lot criticism from more applied research due to its inability including more complex aspects of transport, such as cost, time, strategies, and scenarios, but also graphical aspects. Indeed, a recurrent problem of transport networks is their visualization and cartography. Recent advances in graph visualization algorithms have provided certain solutions to represent, for instance, airline networks as planar networks using so-called edge-bundling tools, in order to limit the fuzziness of mappings caused by the intermingling of numerous, space-constrained links and nodes in a large transport network (Lambert et al., 2013). In turn, exploring the multifaceted dimension of transport networks, such as their territorial embedding, had mobilized other methodologies than sole graph theory. One good example is the raster-based analysis of global urban accessibility taking into account no less than ten different layers in a GIS understood as "friction surface components" (Nelson, 2008). Despite its fundamental graph-theoretical essence, a work on the road accessibility of European seaports integrated several qualitative aspects such as national regulations in terms of speed limits and resting times for truckers, the presence and cost of highway tolls, the types and capacities of main road arteries, and the loading/unloading potential delays at intermodal junctions (Chapelon, 2006). In the case of urban streets, more qualitative aspects were introduced by "space syntax", which proposed to define links based on the continuity of avenues and 
boulevards from a cognitive point of view instead of being alignments of arbitrarily separated segments (Hillier and Hanson, 1984). Such an approach could, by transforming lines into nodes and nodes into lines, reveal hidden dimensions of the network, the hierarchy of streets, which was not visible via the more traditional, planar perspective. Many measures did not provide extraordinary new results, since in general, the traffic volume of transport nodes, which is known from classic sources, is highly equivalent to any centrality measure: large seaports or seaports have many links and vice-versa. Rare studies, however, have truly investigated the spatial determinants of the centrality of transport nodes. These include statistical evidence about the role of urban and regional socio-economic characteristics mostly in the realm of airline networks, where cities are nodes and aircraft trips are links. Main results point at noticeable interdependencies between centrality and the well-being of localities. At least could have been made evident certain linkages between urban indicators and network indicators for cities such as in Europe based on airline data, often concluding to a circular causation between urban and transport development. The spatial dimension was also included, perhaps more by physicists than by geographers, so as to demonstrate rather trivial, albeit fundamental, properties of transport networks whereby larger nodes connect more distant nodes on average. Such works shared some affinities with the regional science perspective on transport hubs where cost and time are central parameters.

Given such limitations and despite the improvements brought by GIS-T and natural sciences, geography and other social sciences have increasingly defined transport networks as elements of wider structures. The shift from quantitative/structural to more behavioral approaches is one main explanation (Waters, 2006), but also the fact that network analysis mostly considers transport networks as simplified and self-sufficient entities developed in 
abstract spaces. New concepts that emerged in the late 1990s, such as global production networks, value and commodity chains, have in common a disregard of pure physical flows, more concerned by the "flesh" than the "bones" when dealing with actors, mobility, governance, and territorial issues at various scales. A transport network can thus be approached via more complex and parent configurations, planned or not planned, such as corridors and gateways, although the two latter concepts are often subcomponents of the transport network itself.

Another way to place transport networks into a wider analytical perspective has been proposed by urban geographers, in implicit or explicit ways. Early urban models, such as the central place theory and up to the New Economic Geography (NEG), approached transport networks in a rather abstract fashion while conferring them vital importance in the emergence of cities and hubs within and between regions. Nevertheless, most NEG studies keep considering cities and regions as equally spaced, thus somewhat excluding the friction exerted by transport networks on mobility. Studies of urban systems and systems of cities were more keen on analyzing urban development through the more concrete configuration of transport networks, such as railway networks. In such a perspective, the transport network is used as a proxy for and a material illustration of - wider inter-urban interactions and dynamics in terms of spatial expansion, functional diversification, hierarchical transformations, and innovation cycles. Simulation methods are used to extract meaningful properties about both transport and urban dynamics and to confront estimates with real population data across many decades or centuries. Yet, there are very rare attempts to integrate urban or regional attributes into transport network analysis and/or modeling, such as the one of Schintler et al. (2007) analyzing the vulnerability of road and railway networks in Florida through a mixture of various methods and approaches. 
More efforts are thus need to push further this integration in order to better meet current challenges in transport and society.

SEE ALSO: GIS-T; Graph Theory; Maritime Transport; Network Analysis; New Economic Geography; Rail Transport (Freight); Rail Transport (Passengers); Road Transport; Routing and Navigation; Transport Geography; Transport and Transportation Systems; Visualization

\section{References and Further Readings (9 max.)}

Barthelemy, M. (2011). Spatial networks. Physics Reports, 499, 1-101.

Barthélemy, M. \& Flammini, A. (2009). Co-evolution of density and topology in a simple model of city formation. Networks and Spatial Economics, 9(3), 401-425.

Bogart, D. (2009). Inter-modal network externalities and transport development: Evidence from roads, canals, and ports during the English Industrial Revolution. Networks and Spatial Economics, 9(3), 309-338.

Ducruet, C. \& Lugo I. (2013). Structure and dynamics of transport networks: Models, methods, and applications. In J. P. Rodrigue, T. E. Notteboom and J. Shaw (Eds.), The SAGE handbook of transport studies (pp. 347-364). London: SAGE Publications Ltd.

Hillier, B. \& Hanson, J. (1984). The social logic of space. Cambridge: Cambridge University Press.

Kansky, K. J. (1963). The structure of transportation networks: Relationships between network geography and regional characteristics (Research Paper No. 84). Chicago: University of Chicago.

Lambert, A., Bourqui, R. \& Auber, D. (2013). Graph visualization for geography. In C. Rozenblat and G. Melançon (Eds.), Methods for multilevel analysis and visualisation of geographical networks (pp. 81-102). Springer.

Nelson, A. (2008). Estimated travel time to the nearest city of 50,000 or more people in year 2000. Global Environment Monitoring Unit - Joint Research Centre of the European Commission, Ispra Italy.

Newman, M. E. J. (2010). Networks: an introduction. New York: Oxford University Press.

Rodrigue, J. P. (2014) Conceptual corridor development. The Geography of Transport Systems, https://people.hofstra.edu/geotrans/eng/ch2en/conc2en/corridordev.html 
Schintler, L. A., Kulkarni, R., Gorman, S. \& Stough, R. (2007). Using raster-based GIS and graph theory to analyze complex networks. Networks and Spatial Economics, 7(4), 301-313.

Taaffe, E. J. \& Gauthier, H. L. (1973). Geography of transportation. Englewood Cliffs, NJ, USA: Prentice-Hall.

Tero, A., Takagi, S., Saigusa, T., Ito, K., Bebber, D. P., Fricker, M. D., Yumiki, K., Kobayashi, R. \& T. Nakagaki (2010). Rules for biologically inspired adaptive network design. Science, 327(5964), 439-442.

Waters, N. (2006). Network and nodal indices. Measures of complexity and redundancy: A review. In A. Reggiani and P. Nijkamp (Eds.), Spatial dynamics, networks and modelling (pp. 13-33). Northampton: Edward Elgar Publishing.

Xie, F. \& Levinson, D. (2009). Modeling the growth of transportation networks: A comprehensive review. Networks and Spatial Economics, 9(3), 291-307.

\section{Key Words}

Networks; Spatial Analysis; Transportation 